\title{
Abnormal immunothrombosis and lupus anticoagulant in a catastrophic COVID-19 recalling Asherson's syndrome
}

\author{
Luca Roncati $^{1}$ [ - Lorenzo Corsi ${ }^{2} \cdot$ Giuseppe Barbolini $^{1}$ \\ Accepted: 30 March 2021 / Published online: 12 April 2021 \\ (c) The Author(s), under exclusive licence to Springer Science+Business Media, LLC, part of Springer Nature 2021
}

\begin{abstract}
Background Coronavirus disease 2019 (COVID-19) is a complex disease with many clinicopathological aspects, including abnormal immunothrombosis, and the full comprehension of its pathogenetic mechanisms is urgently required.

Methods/Results By means of a multidisciplinary approach, we here report a catastrophic COVID-19 in a 44-year-old Philippine male patient, discovered lupus anticoagulant (LAC)-positive shortly before death, occurred 8 days after hospitalization in a clinical scenario refractory to standard high acuity care recalling Asherson's syndrome (catastrophic antiphospholipid syndrome).

Conclusion A parallelism between this severe form of COVID-19 and Asherson's syndrome can be so drawn. Both the diseases in fact exhibit hypercytokinemia, thrombotic microangiopathy, disseminated intravascular coagulation and multiple organ failure, they show a relationship with viral infections, and they are burdened by a high mortality rate. A genetic predisposition to develop these two overlapping conditions may be supposed.
\end{abstract}

Keywords Coronavirus disease 2019 (COVID-19) · Abnormal immunothrombosis · Lupus anticoagulant (LAC) . Megakaryocytes · Antiphospholipid syndrome (Hughes syndrome) · Asherson's syndrome

\section{Highlights}

- COVID-19 is the most dramatic pandemic of the new millennium characterized by many clinicopathological facets, among which abnormal immunothrombosis

- Abnormal immunothrombosis is a leading cause of COVID-19 related deaths, and its impact can be amplified by the presence of antiphospholipid autoantibodies, such as LAC

- In this setting, the simultaneous occurrence of venous and arterial thromboses should alert the clinicians

Luca Roncati

luca.roncati@unimore.it; roncati.luca@aou.mo.it;

emailmedical@gmail.com

1 Department of Surgery, Medicine Dentistry and Morphological Sciences with Interest in Transplantation, Oncology and Regenerative Medicine, Institute of Pathology, University of Modena and Reggio Emilia, Polyclinic Hospital, Largo del Pozzo 71, 41124 Modena (MO), Italy

2 Department of Life Sciences, Section of Pharmacology, University of Modena and Reggio Emilia, Modena, Italy towards a secondary antiphospholipid syndrome, sometimes burdened by a catastrophic evolution in genetically predisposed patients

- The catastrophic variant of a secondary antiphospholipid syndrome takes also the name of Asherson's syndrome, from the rheumatologist Ronald Andrew Asherson, who described it in 1992

- The implications of all this for future directions are in favor of a routine antiphospholipid testing in severe COVID-19 patients, and its introduction under intensive care as potential prognostic risk marker

\section{Introduction}

As well known, thrombosis represents a pathological event inside intact blood vessels; however, immunothrombosis has been reconsidered in the last few years, since it can locally confine an infection through a synergistic action among platelets, coagulation factors, fibrin, inflammatory cells, and neutrophil extracellular traps [1]. In case of imbalance, immunothrombosis can rapidly become abnormal and to evolve into disseminated intravascular coagulation (DIC) 
[2]. Coronavirus disease 2019 (COVID-19) is a complex disease with many clinicopathological issues, including respiratory, immune, and coagulative ones [3-5]. Lupus anticoagulant (LAC) is an antiphospholipid (aPL) autoantibody, which in vivo works as procoagulant, despite the misnomer due to its in vitro properties, by interacting with platelet and endothelial membrane phospholipids, so rising adhesion and aggregation of platelets [6]. Several viral infections run with aPL antibodies production (LAC, anti-cardiolipin, antiapolipoprotein) and, in critically ill patients treated under intensive care, alongside the frequent LAC development, there is also a higher density of lung-resident megakaryocytes $[7,8]$.

\section{Report}

On minimally invasive post-mortem examination as per US Centers for Disease Control and Prevention's guidelines, we have disclosed one-order-of-magnitude increase of nakedmegakaryocyte-nuclei (NK-MK), i.e. $100-140 \mathrm{~cm}^{2}$ versus 14-15 $\mathrm{cm}^{2}$ in normal conditions [5], inside the damaged lungs of a 44-year-old Philippine male COVID-19 patient, discovered LAC-positive shortly before death [silica clotting time (SCT) screen \& confirm: 1,68 \& 1,79; diluted Russell's viper venom test (DRVVT) screen \& confirm: $2,07 \& 1,15$; normal ratios: $\leq 1,20]$, and died 8 days after hospitalization in a clinical scenario refractory to standard high acuity care recalling Asherson's syndrome, also named «catastrophic antiphospholipid syndrome» (Fig. 1). In the patient's medical history, the only disorder worthy of note was type II diabetes mellitus under dietary control. In brief, following the onset of a feverish state with dyspnea, dry cough, sore throat, headache and diarrhea, the patient went to the emergency room, from which he was admitted to the infectious disease ward for COVID-19 pneumonia, as confirmed by imaging (Fig. 1a, b) and molecular nasopharyngeal swab. Shortly thereafter, an episode of peripheral desaturation occurred, for which the patient was subjected to oxygen therapy plus antibiotic coverage (azithromycin), antipyretic (paracetamol), hydration and insulin therapy; corticosteroids were avoided due to the underlying diabetes. Nevertheless, on the third day from admission, the patient became hemodynamically unstable and an arterial blood gas analysis revealed severe hypoxemia $\left(\mathrm{P}_{\mathrm{a}} \mathrm{O}_{2}<40 \mathrm{mmHg}\right)$, so the patient in acute respiratory distress was transferred to the intensive care unit, where he was sedated, intubated and submitted to mechanical ventilation by continuous positive airway pressure. On the same day, he developed a left testicular swelling, and a venous sample for LAC test was taken in the suspicion of a thrombotic event. The result of LAC positivity was made available only the day before death, when the therapeutic introduction of heparin in continuous infusion was adopted. Blood tests on the death day showed: white blood cells count $32.80 \times 10^{3} \mu \mathrm{L}$ including $29.82 \times 10^{3} \mu \mathrm{L}$ neutrophils, platelets $501 \times 10^{3} \mu \mathrm{L}$, D-dimer $2.390 \eta \mathrm{g} / \mathrm{mL}$, fibrinogen $786 \mathrm{mg} / \mathrm{dL}$, antithrombin $46 \%$, activated partial thromboplastin time ratio 2,03 , glucose $170 \mathrm{mg} / \mathrm{dL}$, procalcitonin $0,9 \eta \mathrm{g} / \mathrm{mL}$, B-type natriuretic peptide $281 \mathrm{\rho g} / \mathrm{mL}$, troponin $11.200 \eta \mathrm{g} / \mathrm{L}$, lactate dehydrogenase $723 \mathrm{U} / \mathrm{L}$, creatine kinase $883 \mathrm{U} / \mathrm{L}$, lipase $206 \mathrm{U} / \mathrm{L}$, albumin $2.3 \mathrm{~g} / \mathrm{dL}$, sodium $150 \mathrm{mmol} / \mathrm{L}$, potassium $7.3 \mathrm{mmol} / \mathrm{L}$, calcium $0.96 \mathrm{mmol} / \mathrm{L}$, chlorine $114 \mathrm{mmol} / \mathrm{L}$, magnesium $3.0 \mathrm{mg} / \mathrm{dL}$, urea $79 \mathrm{mg} /$ $\mathrm{dL}$, creatinine $4.97 \mathrm{mg} / \mathrm{dL}$, and an estimated glomerular filtration rate at $13 \mathrm{~mL} / \mathrm{min}$; for all this, the patient devoid of conscience was placed in continuous venovenous hemofiltration $(\mathrm{CVVH})$ without success. Unfortunately, there was no time to combine CVVH with plasmapheresis.

\section{Discussion}

The numerous NK-MK found on post-mortem lung specimens are maybe related to high interleukin-6 (IL-6) serum levels $(>2.300 \rho \mathrm{g} / \mathrm{mL}$ ) from cytokine storm stimulating pulmonary megakaryocytopoiesis, being IL-6 endowed of megakaryocytopoietic activity [5]. NK-MK in fact mean megakaryocytes cytoplasm has been consumed to produce neo-platelets, then released when still alive in the lung microcirculation with prothrombotic effects, which can be dramatically amplified by LAC. In accordance with the emerging evidence of aPL antibodies in COVID-19 [9], we here support a real LAC role in abnormal immunothrombosis and coagulopathy in a life-threatening subgroup of COVID-19 patients, advancing LAC test as potential prognostic risk marker under intensive care in COVID-19. In this setting, the simultaneous occurrence of venous and arterial thromboses, as reported in $3.7 \%$ of COVID-19 patients [10], should alert the clinicians towards a secondary aPL syndrome, whose catastrophic variant is precisely defined Asherson's syndrome from the rheumatologist Ronald Andrew Asherson [Dec 19, 1934, Cape Town (ZA)-May 06, 2008, Johannesburg (ZA)], who described the syndrome that bears his name in 1992 [11]. It exhibits hypercytokinemia, thrombotic microangiopathy, DIC and multiple organ failure, exactly as observed in our patient, it is burdened by a high mortality rate and, although its cause remains still unknown, viral infections have been identified like trigger factors in certain individuals with genetic predisposition correlated to human leukocyte antigen (HLA) polymorphisms (HLA-DR4, HLADR7, HLA-DRw53) [12]. Therefore, a routine testing for aPL antibodies in severe COVID-19 patients is recommended. 

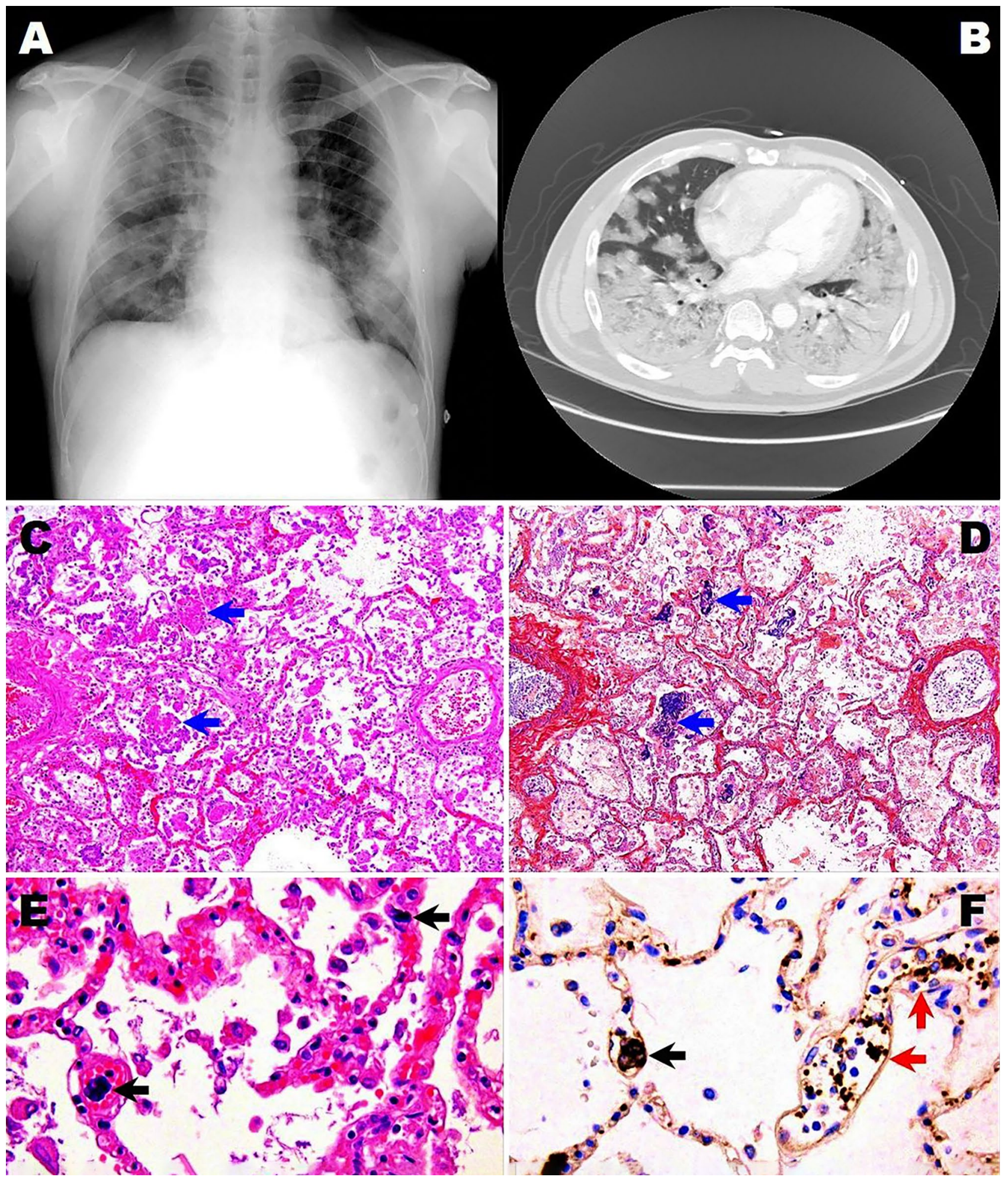

Fig. 1 Chest X-ray upon admission to hospital (a) and lungs CT axial scan the next day (b) showing massive COVID-19 pneumonia in a 44-year-old Philippine male patient, discovered LAC-positive shortly before death, occurred 8 days after hospitalization in a clinical scenario refractory to standard high acuity care recalling Asherson's syndrome: on post-mortem lung histopathology, hyaline membranes, the hallmark of diffuse alveolar damage in exudative phase, are detected by hematoxylin $\&$ eosin staining (c, blue arrows, $\times 10$ objective), and, in the same field, phosphotungstic acid hematoxylin stains in dark blue the hyaline membranes' fibrin (d, blue arrows, $\times 10$ objective). At higher magnification, inside the interstitial capillaries, NK-MK are well noticeable by hematoxylin $\&$ eosin (e, black arrows, $\times 40$ objective); immunohistochemistry for CD61 (f, 2 f2 clone, $\times 40$ objective) specifically labels in brown, through 3,3'-diaminobenzidine as chromogen, both NK-MK (f, black arrow, $\times 40$ objective) and neo-platelets which clog microcirculation (f, red arrows, $\times 40$ objective) 
Funding This research received no external funding.

Data availability The data presented in this study are available on reasonable request from the corresponding author; the data are not publicly available due to privacy or ethical restrictions.

\section{Declarations}

Conflict of interest The authors declare no conflict of interest.

Ethical approval All procedures followed were in accordance with the ethical standards and with the Helsinki Declaration of 1975, as revised in 2008 .

Informed consent Not applicable since the patient died.

\section{References}

1. Grover SP, Mackman N (2018) Neutrophils, NETs, and immunothrombosis. Blood 132:1360-1361

2. Gaertner F, Massberg S (2016) Blood coagulation in immunothrombosis: at the frontline of intravascular immunity. Semin Immunol 28:561-569

3. Roncati L, Nasillo V, Lusenti B, Riva G (2020) Signals of $T_{h} 2$ immune response from COVID-19 patients requiring intensive care. Ann Hematol 99:1419-1420

4. Roncati L, Gallo G, Manenti A, Palmieri B (2020) Renin-angiotensin system: the unexpected flaw inside the human immune system revealed by SARS-CoV-2. Med Hypotheses 140:109686

5. Roncati L, Ligabue G, Nasillo V, Lusenti B, Gennari W, Fabbiani L, Malagoli C, Gallo G, Giovanella S, Lupi M, Salviato T, Paolini
A, Costantini M, Trenti T, Maiorana A (2020) A proof of evidence supporting abnormal immunothrombosis in severe COVID-19: naked megakaryocyte nuclei increase in the bone marrow and lungs of critically ill patients. Platelets 31:1085-1089

6. Ruiz-Irastorza G, Crowther M, Branch W, Khamashta MA (2010) Antiphospholipid syndrome. Lancet 376:1498-1509

7. Abdel-Wahab N, Talathi S, Lopez-Olivo MA, Suarez-Almazor ME (2018) Risk of developing antiphospholipid antibodies following viral infection: a systematic review and meta-analysis. Lupus 27:572-583

8. Mandal RV, Mark EJ, Kradin RL (2007) Megakaryocytes and platelet homeostasis in diffuse alveolar damage. Exp Mol Pathol 83:327-331

9. Roncati L, Manenti A, Manco G, Farinetti A, Mattioli A (2020) The Covid-19 arterial thromboembolic complications: from inflammation to immunothrombosis through anti-phospholipid autoantibodies. Ann Vasc Surg. https://doi.org/10.1016/j.avsg. 2020.12.006

10. Klok FA, Kruip MJHA, van der Meer NJM, Arbous MS, Gommers DAMPJ, Kant KM, Kaptein FHJ, van Paassen J, Stals MAM, Huisman MV, Endeman H (2020) Incidence of thrombotic complications in critically ill ICU patients with COVID-19. Thromb Res 191:145-147

11. Asherson RA (1992) The catastrophic antiphospholipid syndrome. J Rheumatol 19:508-512

12. Iuliano A, Galeazzi M, Sebastiani GD (2019) Antiphospholipid syndrome's genetic and epigenetic aspects. Autoimmun Rev 18:102352

Publisher's Note Springer Nature remains neutral with regard to jurisdictional claims in published maps and institutional affiliations. 